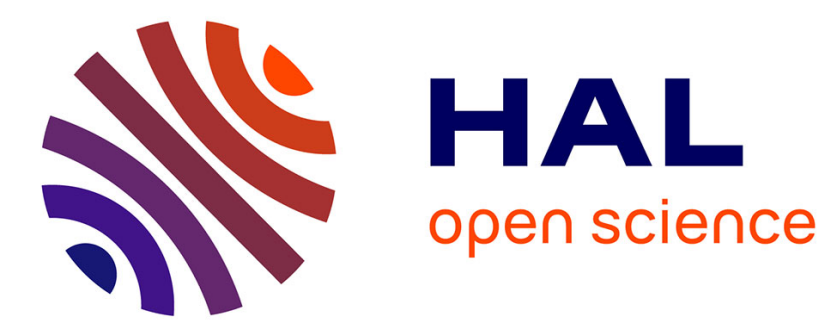

\title{
About the synchronization of MEMs
}

Jean Lerbet

\section{To cite this version:}

Jean Lerbet. About the synchronization of MEMs. Nonlinear Analysis: Real World Applications, 2009, 10 (1), pp.266-276. 10.1016/j.nonrwa.2007.09.009 . hal-00342814

\section{HAL Id: hal-00342814 https://hal.science/hal-00342814}

Submitted on 13 Nov 2019

HAL is a multi-disciplinary open access archive for the deposit and dissemination of scientific research documents, whether they are published or not. The documents may come from teaching and research institutions in France or abroad, or from public or private research centers.
L'archive ouverte pluridisciplinaire HAL, est destinée au dépôt et à la diffusion de documents scientifiques de niveau recherche, publiés ou non, émanant des établissements d'enseignement et de recherche français ou étrangers, des laboratoires publics ou privés. 


\title{
About the synchronization of MEMs
}

\author{
Jean Lerbet* \\ IBISC, FRE CNRS 2873, Universit'e d'Evry Val d'Essone, 40 Rue du Pelvoux, CE 1455 Courcouronnes, 91020 Evry Cedex,
}

France

\begin{abstract}
The stabilization of motions of MEMs is a difficult and important problem for the users because of the many nonlinearities of the system and the advantage for the structure to work close to resonance for a better electro-mechanical coupling. In this paper, we investigate the synchronized motion of a one degree of freedom model of MEMs taking into account the nonlinear effect of the electric force. By using the theory of synchronization with $\mu=\frac{\delta V}{V_{e}}$ as small parameter, we propose a procedure to ensure the synchronized motion close to resonance and to investigate its asymptotic stability. To our knowledge, this problem has never been investigated.
\end{abstract}

Keywords: MEMs; Synchronization; Small parameter; Nonlinear effects

\section{Introduction}

For many years, several modeling of MEMs have been proposed and specific procedures are now programmed with softwares like ANSYS. Nonlinear effects (geometry, electrostatic forces ...) are taken into account and coupled electo-mechanical calculations are also proposed. As noted in [7], the situation is not so clear concerning dynamic calculations and several investigations have to be carried out, for example about damping or dynamic electromechanical coupling effects. Dynamics of nonlinear systems may have complicated behavior from regular to chaotic one with every kind of instability (divergence, flutter, Hopf bifurcations, limit cycles ...) (see $[4,6,8,13,9]$, etc). Moreover, the MEMs are used close to resonance because of a better electro-mechanical coupling. However, that may induce the collapse or pull-in and the control of motion close to resonance is in fact one of the final aims in using MEMs. We propose here to contribute to such an aim by applying the theory of synchronization to the dynamics of MEMs. In order to present the essence of the method, we only use a one degree of freedom equivalent system. The limitations of such a model are well-known but we think that the higher forgetting lies in a frequent assumption consisting in supposing that the electrostatic force is "vertical" and is not a real pression. However, nonlinear nonuniform pression may induce effects like those induced by a follower force. Such effects are (often and here by nature of a one d.o.f. system) neglected and we focus only on nonlinear effects. Remark nevertheless that without neglecting the previously mentioned effects, static instability (divergence) (as pull-in is often presented) is

\footnotetext{
* Corresponding address: Departement of Mechanical Engineering, Universite d'Evry Val d'Essone, 40 Rue du Pelvoux, CE1455 Courcouronnes, 91020 Evry Cedex, France.

E-mail address: jean.lerbet@ibisc.univ-evry.fr.
} 


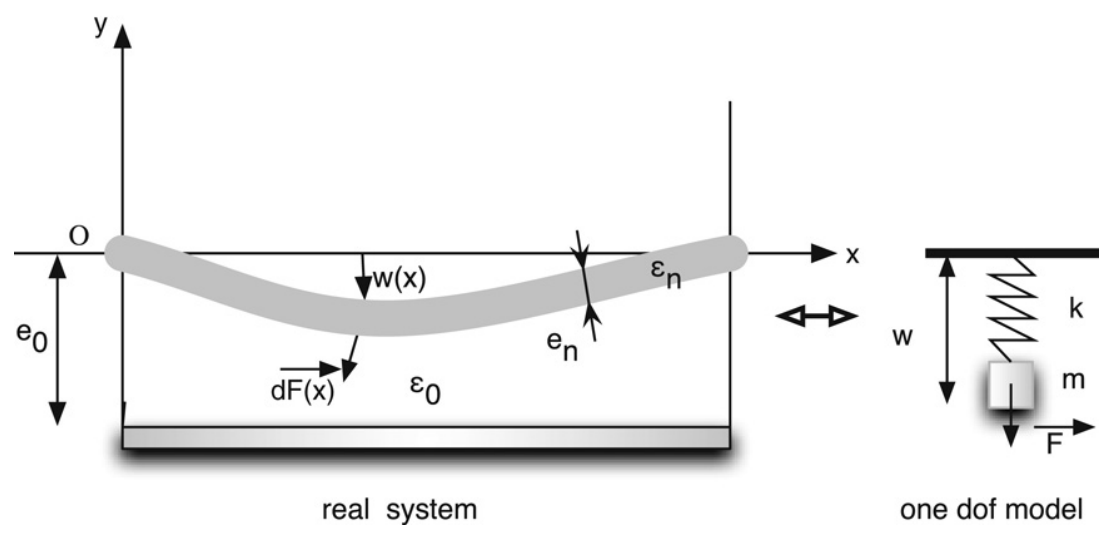

Fig. 1. The model.

not identical to the dynamic one (flutter) $([3,5,1], \ldots)$. That is also mentioned in [10] after simulations. The author mentions "that the system may become unstable before the static pull-in voltage due to the dynamical effect and thus one should consider the dynamic pull-in voltage".

In the first section the mechanical model is presented. The second section is devoted to the derivation of synchronization's equations. In the last part, MAPLE procedure allowing numerical simulations and experimental verifications are given.

\section{The model and the equation}

According to the mechanical model, the dynamic equations are:

$$
m \ddot{v}+k v=F(v, V)
$$

where $m$ is the equivalent mass and $k$ is the equivalent rigidity of the system. $F(v, V)$ denotes the electric force acting on the mass when a voltage $V$ is applied and (see [2] for example):

$$
F(v, V)=\frac{a V^{2}}{(1+b(1-v))^{2}}=V^{2} H(v)
$$

with $a, b$ parameters depending on geometrical and physical data. This expression means that fringing effects are here neglected [12]. Because $V$ is supposed not depending on $v$, coupling effects are neglected too. $m$ and $k$ are scalars containing as much as possible information about the real system and

$$
H(v)=\frac{a}{(1+b(1-v))^{2}}
$$

For example, they are obtained after using of Rayleigh-Ritz Method and may take into account the boundary conditions. More precisely, we have got:

$$
\begin{aligned}
& a=\frac{\epsilon_{n}^{2} A}{2 e_{0} \epsilon_{0}^{2} e_{n}^{2}}=\frac{\epsilon_{0}{\tilde{\epsilon_{n}}}^{2} A}{2 e_{0} e_{n}^{2}} \\
& b=\frac{\epsilon_{n} e_{0}}{\epsilon_{0} e_{n}}=\frac{\tilde{\epsilon_{n}} e_{0}}{e_{n}}
\end{aligned}
$$

where $\epsilon_{0}$ is the dielectric constant of vacuum, $\epsilon_{n}$ is the dielectric constant of the matter, $\tilde{\epsilon_{n}}$ the relative dielectric constant of the matter, $e_{0}$ is the gap spacing of the cavity, $e_{n}$ the thickness of the membrane and $A$ the capacitor surface (see Fig. 1). $v=\frac{w}{e_{0}}$ is dimensionless and $w$ is the displacement.

Let us suppose that an equilibrium position $v_{e}$ is reached when the DC voltage $V_{e}$ is applied. This means that:

$$
k v_{e}=F\left(v_{e}, V_{e}\right)=V_{e}^{2} H\left(v_{e}\right) .
$$


In the following, the nonlinearity shall be investigated through a small parameter $\mu$ defined by:

$$
V=V_{e}(1+\mu \sin (\omega t)) \quad \text { or } \quad \mu=\frac{\delta V}{V_{e}}
$$

where $\delta V$ is the amplitude of additional AC voltage. Consider $u=v-v_{e}$. (1) becomes successively by using (5), considering $u$ as a small quantity and neglecting the terms containing $\mu^{2}$ :

$$
\begin{aligned}
m \ddot{u}+k u & =V^{2} H\left(u+v_{e}\right)-k v_{e} \\
& =V_{e}^{2}(1+\mu \sin (\omega t))^{2} H\left(u+v_{e}\right)-k v_{e} \\
& \approx V_{e}^{2} H\left(u+v_{e}\right)+2 \mu \sin (\omega t) H\left(u+v_{e}\right)-V_{e}^{2} H\left(v_{e}\right) \\
& \approx V_{e}^{2}\left(H\left(v_{e}\right)+u H^{\prime}\left(v_{e}\right)\right)+2 \mu \sin (\omega t) H\left(u+v_{e}\right)-V_{e}^{2} H\left(v_{e}\right) \\
& \approx V_{e}^{2} u H^{\prime}\left(v_{e}\right)+2 \mu \sin (\omega t) V_{e}^{2} H\left(u+v_{e}\right) .
\end{aligned}
$$

This leads to the following equation:

$$
\ddot{u}+\omega_{1}^{2} u=\mu h(u, \omega t)
$$

where

$$
\begin{aligned}
\omega_{1}^{2} & =\frac{k-V_{e}^{2} H^{\prime}\left(v_{e}\right)}{m} \\
& =\frac{V_{e}^{2}}{m}\left(\frac{H\left(v_{e}\right)}{v_{e}}-H^{\prime}\left(v_{e}\right)\right)
\end{aligned}
$$

and

$$
h(u, \psi)=2 \frac{V_{e}^{2}}{m} H\left(\left(v_{e}+u\right) \sin (\psi)\right) .
$$

In the relation (7) appears the so-called "softening" of the structure, a phenomenon well-known in the community of MEMs. $\theta$ defined by: We shall now investigate the solution of (6) and more precisely the possibility of a periodic motion.

\section{Synchronization}

We recall now the fundamental result concerning the synchronization and we apply it to our problem. Our reference is [11].

\subsection{Synchronized motion and its stability}

Let us consider the following differential system:

$$
\left\{\begin{array}{l}
\frac{\mathrm{d} x}{\mathrm{~d} t}=A x+\mu f(x, y, \mu, t) \\
\frac{\mathrm{d} y}{\mathrm{~d} t}=\mu g(x, y, \mu, t)
\end{array}\right.
$$

where $x, y$ are vectorial n-dimensional and p-dimensional variables, $A$ an $n \times n$ real constant matrix. Suppose that $f: U \times V \times I \times \mathbb{R} \rightarrow \mathbb{R}^{n}$ and $g: U \times V \times I \times \mathbb{R} \rightarrow \mathbb{R}^{p}$ are continuous, $\mathcal{C}^{1}$ with respect to $x, y, \mu, T$-periodic as function of $t, U$ an open of $\mathbb{R}^{n}$ containing $0, V$ an open of $\mathbb{R}^{p}$ containing $y_{0}$ such that

$$
G\left(0, y_{0}\right)=0
$$

with

$$
G(x, y)=\frac{1}{T} \int_{0}^{T} g(x, y, 0, t) \mathrm{d} t
$$


with $I=\left[0, \mu_{0}\right]$. If

$$
P\left(y_{0}, t\right)=\frac{\partial g}{\partial y}\left(0, y_{0}, 0, t\right)
$$

and if

$$
S=S\left(y_{0}\right)=\frac{1}{T} \int_{0}^{T} P\left(y_{0}, t\right) \mathrm{d} t
$$

then the following result holds: Fundamental Theorem

If $S$ is a nonsingular matrix, if $\frac{\mathrm{d} x}{\mathrm{~d} t}=A x$ has no $T$-periodic solution, then the system (9) has got for sufficiently small $\mu$, a $T$-periodic solution, that for $\mu \rightarrow 0$ tends towards the solution $x=0, y=y_{0}$. Moreover, if the proper values of $S$ and $A$ have got negative real parts, this periodic solution is asymptotic stable for $t \rightarrow \infty, \mu>0$.

(see [11] pp. 242)

The goal of the following subsection is to transform (6) into a system similar to (9) and to explicit Eq. (10) for our problem.

\subsection{Obtention of a system similar to (9)}

Put:

$$
\omega_{1}^{2}=\omega^{2}(1+\mu \eta)
$$

which defines the number $\eta$. The principle of the method consists in a change of unknowns allowing the modulation of amplitude of the response. Let $y$ and $z$ be two new unknowns such that

$$
u=y \cos (z+\phi)
$$

where the new variable $\phi$ is given by

$$
\phi=\omega t .
$$

We obtain

$$
\frac{\mathrm{d} u}{\mathrm{~d} \phi}=-y \sin (z+\phi)
$$

if the following additional condition holds

$$
\frac{\mathrm{d} y}{\mathrm{~d} \phi} \cos (z+\phi)-y \frac{\mathrm{d} z}{\mathrm{~d} \phi} \sin (z+\phi)=0
$$

(6) is then successively equivalent to:

$$
\begin{aligned}
\frac{\mathrm{d}^{2} u}{\mathrm{~d} \phi^{2}} & =-\frac{\omega_{1}^{2}}{\omega^{2}} u+\frac{1}{\omega^{2}} \mu h(u, \phi) \\
& =-(1+\mu \eta) u+\frac{1}{\omega^{2}} \mu h(u, \phi) \\
& =-u+\mu\left(-\eta u+\frac{1}{\omega^{2}} h(u, \phi)\right)
\end{aligned}
$$

(15) gives by differentiation:

$$
\frac{\mathrm{d}^{2} u}{\mathrm{~d} \phi^{2}}=-\frac{\mathrm{d} y}{\mathrm{~d} \phi} \sin (z+\phi)-y \cos (z+\phi)-y \frac{\mathrm{d} z}{\mathrm{~d} \phi} \cos (z+\phi)
$$

or by using (17) and (13):

$$
-\frac{\mathrm{d} y}{\mathrm{~d} \phi} \sin (z+\phi)-y \frac{\mathrm{d} z}{\mathrm{~d} \phi} \cos (z+\phi)=\mu\left(-\eta y \cos (z+\phi)+\frac{1}{\omega^{2}} h(y \cos (z+\phi), \phi)\right)
$$


(16) and (18) lead after multiplying conveniently by $\sin (z+\phi)$ and $\cos (z+\phi)$ and after addition and subtraction to:

$$
\left\{\begin{array}{l}
\frac{\mathrm{d} y}{\mathrm{~d} \phi}=\mu \sin (z+\phi) g(y, z, \mu, \phi) \\
\frac{\mathrm{d} z}{\mathrm{~d} \phi}=\frac{\mu}{y} \cos (z+\phi) g(y, z, \mu, \phi)
\end{array}\right.
$$

with

$$
\begin{aligned}
g(y, z, \mu, \phi) & =\eta y \cos (z+\phi)-\frac{1}{\omega^{2}} h(y \cos (z+\phi), \phi) \\
& =\eta y \cos (z+\phi)-\frac{1+\mu \eta}{\omega_{1}^{2}} h(y \cos (z+\phi), \phi) .
\end{aligned}
$$

System (19) is strictly similar to (9) with the following identifications:

$$
\begin{aligned}
& n=0, \quad p=2, \quad y=(y, z), \quad \omega t=\phi, \\
& T=\frac{2 \pi}{\omega}, \quad g(x, y, \mu, t)=(\sin (z+\phi) g(y, z, \mu, \phi), \cos (z+\phi) g(y, z, \mu, \phi))
\end{aligned}
$$

where $g(y, z, \mu, \phi)$ is given by (20).

\subsection{Obtention of equations of synchronization equivalent to (10)}

According to the theory of synchronization, consider now both the following average quantities that must vanish:

$$
\begin{aligned}
& Y(y, z)=\frac{1}{2 \pi} \int_{0}^{2 \pi} \sin (z+\phi) g(y, z, 0, \phi) \mathrm{d} \phi \\
& Z(y, z)=\frac{1}{2 \pi} \int_{0}^{2 \pi} \cos (z+\phi) g(y, z, 0, \phi) \mathrm{d} \phi .
\end{aligned}
$$

Calculations give:

$$
\begin{aligned}
& Y(y, z)=-\frac{1}{2 \pi \omega_{1}^{2}} \int_{0}^{2 \pi} \sin (z+\phi) h(y \cos (z+\phi), \phi) \mathrm{d} \phi \\
& Z(y, z)=\frac{\eta}{2}-\frac{1}{2 \pi \omega_{1}^{2} y} \int_{0}^{2 \pi} \cos (z+\phi) h(y \cos (z+\phi), \phi) \mathrm{d} \phi .
\end{aligned}
$$

Equations of synchronization being $Y(y, z)=Z(y, z)=0$ we have to compute functions $Y$ and $Z$. According to previous expression of $h(u, \psi), Y$ and $Z$ may be rewritten as:

$$
\begin{aligned}
& Y(y, z)=-\frac{V_{e}^{2}}{\pi m \omega_{1}^{2}} \int_{0}^{2 \pi} \sin (z+\phi) H\left(v_{e}+y \cos (z+\phi)\right) \sin (\phi) \mathrm{d} \phi \\
& Z(y, z)=\frac{\eta}{2}-\frac{V_{e}^{2}}{\pi m \omega_{1}^{2} y} \int_{0}^{2 \pi} \cos (z+\phi) H\left(v_{e}+y \cos (z+\phi)\right) \sin (\phi) \mathrm{d} \phi .
\end{aligned}
$$

Using that

$$
\omega_{1}^{2}=\frac{k-V_{e}^{2} H^{\prime}\left(v_{e}\right)}{m}=\frac{V_{e}^{2}}{m}\left(\frac{H\left(v_{e}\right)}{v_{e}}-H^{\prime}\left(v_{e}\right)\right)=\frac{V_{e}^{2}}{m} \Xi\left(v_{e}\right)
$$

with

$$
\Xi(v)=\frac{H(v)}{v}-H^{\prime}(v)
$$


we obtain:

$$
\begin{aligned}
& Y(y, z)=-\frac{1}{\pi \Xi\left(v_{e}\right)} \int_{0}^{2 \pi} \sin (z+\phi) H\left(v_{e}+y \cos (z+\phi)\right) \sin (\phi) \mathrm{d} \phi \\
& Z(y, z)=\frac{\eta}{2}-\frac{1}{\pi \Xi\left(v_{e}\right) y} \int_{0}^{2 \pi} \cos (z+\phi) H\left(v_{e}+y \cos (z+\phi)\right) \sin (\phi) \mathrm{d} \phi
\end{aligned}
$$

or by an obvious change of variables

$$
\begin{aligned}
Y(y, z) & =-\frac{1}{\pi \Xi\left(v_{e}\right)} \int_{z}^{z+2 \pi} \sin (\beta) H\left(v_{e}+y \cos (\beta)\right) \sin (\beta-z) \mathrm{d} \beta \\
& =-\frac{1}{\pi \Xi\left(v_{e}\right)} \int_{z}^{z+2 \pi} \frac{a \sin (\beta) \sin (\beta-z)}{\left(1+b\left(1-v_{e}+y \cos (\beta)\right)\right)^{2}} \mathrm{~d} \beta \\
& =-\frac{a}{\pi b^{2} y^{2} \Xi\left(v_{e}\right)} \int_{z}^{z+2 \pi} \frac{\sin (\beta) \sin (\beta-z)}{(r+\cos (\beta))^{2}} \mathrm{~d} \beta \\
Z(y, z) & =\frac{\eta}{2}-\frac{1}{\pi \Xi\left(v_{e}\right) y} \int_{z}^{z+2 \pi} \frac{a \cos (\beta) \sin (\beta-z)}{\left(1+b\left(1-v_{e}+y \cos (\beta)\right)\right)^{2}} \\
& =\frac{\eta}{2}-\frac{1}{\pi \Xi\left(v_{e}\right) y} \int_{z}^{z+2 \pi} \frac{a \cos (\beta) \sin (\beta-z)}{(a \cos (\beta))^{2}} \mathrm{~d} \beta \\
& =\frac{\eta}{2}-\frac{a}{\pi b^{2} y^{3} \Xi\left(v_{e}\right)} \int_{z}^{z+2 \pi} \frac{\cos (\beta) \sin (\beta-z)}{(r+\cos (\beta))^{2}} \mathrm{~d} \beta
\end{aligned}
$$

where

$$
r=\frac{1}{y}\left(\frac{1}{b}+1-v_{e}\right) .
$$

We then are led to compute integrals like

$$
I(s, r)=\int_{s}^{s+2 \pi} \frac{\cos (\beta) \sin (\beta-s)}{(r+\cos (\beta))^{2}} \mathrm{~d} \beta
$$

and

$$
J(s, r)=\int_{s}^{s+2 \pi} \frac{\sin (\beta) \sin (\beta-s)}{(r+\cos (\beta))^{2}} \mathrm{~d} \beta
$$

or

$$
K(s, r)=I(s, r)+\mathrm{i} J(s, r)=\int_{s}^{s+2 \pi} \frac{\mathrm{e}^{i \beta} \sin (\beta-s)}{(r+\cos (\beta))^{2}} \mathrm{~d} \beta .
$$

An explicit calculation of these quantities is done in the appendix by using Cauchy's Theorem.

Solving these equations, we find $y_{0}=\left(y_{0}, z_{0}\right)$ and coming back to the initial problem (relation (13)), we obtain a $\frac{2 \pi}{\omega}$-periodic solution $u$ of equations of motion (6) close to $u_{0}(t)=y_{0} \cos \left(z_{0}+\omega t\right)$.

We present now Maple Procedures from which the electro-geometrico-mechanical data generate functions $h, H, g$ integrals $I, J$, functions $Y, Z$, compute solution $y_{0}=\left(y_{0}, z_{0}\right)$ of equations of synchronization and generate matrix $S=S\left(y_{0}\right)=S\left(y_{0}, z_{0}\right)$. These procedures allow us to compute periodic motion and investigate its stability.

\section{Maple procedures}

Each procedure corresponds to a variable or a function introduced in the paper by a relation or an equation. It is referenced in each procedure in a comment attached to the procedure. In the first subsection, we present the procedures to generate variables and functions lying in equations of synchronization. In the second subsection, functions generating equations and matrix $S$ are given. 


\subsection{Maple procedures for defining the variables and the functions lying in equations of synchronization}

$>\lambda:=\operatorname{proc}\left(\epsilon_{0}, \epsilon_{n}, e_{0}, e_{n}, A\right) \sharp$ scalar $a$ in the paper according to (3)

$>\epsilon_{0} * \epsilon_{n} * A /\left(2 * e_{0} * e_{n}\right)$;

$>$ end proc;

$>\alpha:=\operatorname{proc}\left(\epsilon_{n}, e_{0}, e_{n}\right) \sharp$ scalar $b$ in the paper according to (4)

$>\left(\epsilon_{n} * e_{0}\right) / e_{n}$

$>$ end proc;

$>H:=\operatorname{proc}(v) \sharp$ function allowing to define the right-hand side of equation according to (2) in the paper

$>$ local $a, b$;

$>$ global $\epsilon_{0}, e_{0}, e_{n}, \epsilon_{n}$

$>a:=\lambda\left(\epsilon_{0}, \epsilon_{n}, e_{0}, e_{n}, A\right)$

$>b:=\alpha\left(\epsilon_{n}, e_{0}, e_{n}\right)$

$>a /(1+b *(1-v))^{2}$;

$>$ end proc;

$>\omega_{1}^{2}:=\operatorname{proc}\left(V_{e}, v_{e}, m\right) \sharp$ according to (7) in the paper

$>$ local $a, b, c, d$;

$>a:=V_{e}^{2} / m$

$>b:=H(v) / v$

$>c:=\operatorname{diff}(H(v), v)$;

$>d:=\operatorname{unapply}(a *(b-c), v)$;

$>d\left(v_{e}\right)$

$>$ end proc;

$>G:=\operatorname{proc}() \sharp$ procedure giving function $\Xi$ of the paper according to (21)

$>$ local $b, c, d$;

$>b:=H(v) / v$

$>c:=\operatorname{diff}(H(v), v)$;

$>d:=\operatorname{unapply}(b-c, v)$

$>$ end proc;

$>h:=\operatorname{proc}(u, \psi) \sharp$ procedure giving function $h(u, \psi)$ of the paper according to (8)

$>$ global $V_{e}, v_{e}, m, \epsilon_{0}, e_{0}, e_{n}, \epsilon_{n}$

$>$ local $a, b$;

$>a:=\lambda\left(\epsilon_{0}, \epsilon_{n}, e_{0}, e_{n}, A\right)$

$>b:=\alpha\left(\epsilon_{n}, e_{0}, e_{n}\right)$

$>2 *\left(V_{e}^{2} / m\right) * H\left(u+v_{e}\right) * \sin (\psi)$;

$>$ end proc;

$>I I:=\operatorname{proc}(s, r) \sharp$ first integral $I(s, r)$ in the paper according to (32)

$>$ local $a$

$>a:=-r+\operatorname{sqrt}\left(r^{2}-1\right)$;

$>(4 * a * p i * \sin (s)) *\left(2 * a+2 * r /\left(r^{2}-1\right)\right) /\left(a^{2}-1\right)$;

$>$ end proc;

$>I J:=\operatorname{proc}(s, r) \sharp$ second integral $J(s, r)$ in the paper according to (33)

$>$ local $a$;

$>a:=-r-\operatorname{sqrt}\left(r^{2}-1\right)$;

$>(4 * p i * \cos (s)) /\left(1-a^{2}\right)$;

$>$ end proc;

\subsection{Equations of synchronization}

Now, we propose procedures for generating equations of synchronization and matrix $S$ for investigating the stability of the motion. $>Y:=\operatorname{proc}(y, z) \sharp$ synchronization: average over a period of first equation according to (22) 
$>$ local $a, b, c, a 1, a 2, a 3$;

$>$ global $V_{e}, v_{e}, \epsilon_{0}, e_{0}, e_{n}, \epsilon_{n}, A$;

$>a:=H(v) / v$;

$>b:=\operatorname{diff}(H(v), v)$

$>c:=$ unapply $(a-b, v)$;

$>a 1:=\lambda\left(\epsilon_{0}, \epsilon_{n}, e_{0}, e_{n}, A\right)$;

$>a 2:=\alpha\left(\epsilon_{n}, e_{0}, e_{n}\right)$

$>a 3:=\left(1 / a 2+1+v_{e}\right) / y$

$>-\left(a 1 /\left(p i * c\left(v_{e}\right) * y^{2} * a 2^{2}\right)\right) * I J(z, a 3)$;

$>$ end proc;

$>Z:=\operatorname{proc}(y, z) \sharp$ synchronization: average over a period of second equation according to (23)

$>$ local $a, b, c, a 1, a 2, a 3$;

$>$ global $\eta, V_{e}, v_{e}, \epsilon_{0}, e_{0}, e_{n}, \epsilon_{n}, A$

$>a:=H(v) / v$

$>b:=\operatorname{diff}(H(v), v)$

$>c:=$ unapply $(a-b, v)$;

$>a 1:=\lambda\left(\epsilon_{0}, \epsilon_{n}, e_{0}, e_{n}, A\right)$;

$>a 2:=\alpha\left(\epsilon_{n}, e_{0}, e_{n}\right)$

$>a 3:=\left(1 / a 2+1+v_{e}\right) / y$

$>(\eta / 2)-\left(a 1 /\left(2 * p i * c\left(v_{e}\right) * y^{3} * a 2^{2}\right)\right) * I I(z, a 3)$

$>$ end proc;

$>S:=\operatorname{proc}() \sharp$ procedure giving the solution $y_{0}=\left(y_{0}, z_{0}\right)$ of equations of synchronization and matrix $S=S\left(y_{0}\right)=$ $S\left(y_{0}, z_{0}\right)$ of synchronization defined by (12)

$>$ local sol, $a, b, g, g 1, g 2, g 3, g 4, g 5, g 6, c$;

$>$ global $Y, Z, h, G, H, v_{e}, \eta$;

$>$ sol := fsolve $(Y(y, z), Z(y, z), y, z)$;

$>a:=\operatorname{eval}(y, \mathrm{sol}) ; \sharp$ First component $y_{0}$ of $y_{0}$

$>b:=\operatorname{eval}(z, \operatorname{sol}) ; \sharp$ Second component $z_{0}$ of $y_{0}$

$>c:=G()\left(v_{e}\right)$

$>g:=$ unapply $\left(\eta * y * \cos (z+\phi)-\left(2 * H\left(v_{e}+y * \cos (z+\phi), \phi\right) / c\right), y, z, \phi\right)$;

$>g 5:=\operatorname{unapply}(\sin (z+\phi) * g(y, z, \phi), y, z, \phi)$;

$>g 6:=\operatorname{unapply}((1 / y) * \cos (z+\phi) * g(y, z, \phi), y, z, \phi)$;

$>g 1:=\operatorname{unapply}(D[1](g 5)(a, b, \phi), \phi)$;

$>g 1:=$ value $(\operatorname{Int}(g 1(\phi), \phi=0 . . P i) /(2 * P i))$;

$>g 1:=\operatorname{evalf}(\%)$

$>g 2:=\operatorname{unapply}(D[2](g 5)(a, b, \phi), \phi)$;

$>g 2:=$ value $(\operatorname{Int}(g 2(\phi), \phi=0 . . P i) /(2 * P i))$;

$>g 2:=\operatorname{evalf}(\%)$

$>g 3:=\operatorname{unapply}(D[1](g 6)(a, b, \phi), \phi)$;

$>g 3:=\operatorname{value}(\operatorname{Int}(g 3(\phi), \phi=0 . . P i) /(2 * P i))$;

$>g 3:=\operatorname{evalf}(\%)$

$>g 4:=\operatorname{unapply}(D[2](g 6)(a, b, \phi), \phi)$;

$>g 4:=\operatorname{value}(\operatorname{Int}(g 4(\phi), \phi=0 . . P i) /(2 * P i)) ;$

$>g 4:=\operatorname{evalf}(\%)$

$>$ Matrix ([[ $g 1, g 2],[g 3, g 4]]) ; \sharp$ Matrix $S$ according to (12)

$>$ end proc;

\section{Conclusion}

This paper is devoted to the investigation of synchronized motion of MEMs close to resonance. This difficult and important problem (for the practice) has never been investigated up to now (to our knowledge). In order to do it, a nonlinear one degree of freedom model has been developed and the theory of synchronization with 
$\mu=\frac{\delta V}{V_{e}}$ as small parameter has been used. Some necessary analytic developments allowing to generate equations of synchronization have been led. To achieve the program, Maple procedures have been given. To use these procedures, equilibrium configuration has to be described $\left(V_{e}, v_{e}\right)$. We think the obtention of these data through the experimental measurements should be better than through the calculations on models because of the other eventual nonlinearities (finite displacements and deformations, nonlinear elasticity). To conclude we have to be precise that the concrete use of MEMs is often made in water which introduces damping in equations, the main result keeping usable.

\section{Acknowledgement}

We would like to thank Pr Jerisian (LMP, Tours University) for introducing this theme to us.

\section{Appendix. Calculation of $K(s, r)$}

\section{Lemma.}

$$
K(s, r)=\frac{2 \mathrm{i} z_{1} \pi}{\left(z_{1}^{2}-1\right)\left(r^{2}-1\right)}\left[\left(2 z_{1}\left(r^{2}-1\right)+r\right) \mathrm{e}^{\mathrm{i} s}-r \mathrm{e}^{-\mathrm{i} s}\right]
$$

where $z_{1}=-r+\sqrt{r^{2}-1}$. We shall use the Cauchy's theorem of residus. Put $z=\mathrm{e}^{\mathrm{i} \beta}$ and let $\gamma$ the circle $|z|=1$. It follows that $\mathrm{d} \beta=-\mathrm{i} \frac{\mathrm{d} z}{z}$ and:

$$
K(s, r)=\int_{\gamma}-\mathrm{i} \frac{z\left(z \mathrm{e}^{-\mathrm{i} s}-\frac{\mathrm{e}^{\mathrm{i} s}}{z}\right)}{2 \mathrm{i}\left(r+\left(\frac{z+\frac{1}{z}}{2}\right)\right)^{2}} \frac{\mathrm{d} z}{z}
$$

or

$$
K(s, r)=\int_{\gamma}-2 \frac{\left(z^{2} \mathrm{e}^{-\mathrm{i} s}-\mathrm{e}^{\mathrm{i} s}\right)}{z\left(z^{2}+2 r z+1\right)^{2}} \mathrm{~d} z
$$

(24) becomes by Cauchy's Theorem:

$$
K(s, r)=-4 \mathrm{i} \pi\left(\operatorname{Res}(0, R)+\operatorname{Res}\left(z_{1}, R\right)\right)
$$

where

$$
R(z)=\frac{\left(z^{2} \mathrm{e}^{-\mathrm{i} s}-\mathrm{e}^{\mathrm{i} s}\right)}{z\left(z^{2}+2 r z+1\right)^{2}}
$$

and $z_{1}$ the root of $z^{2}+2 r z+1$ such that $\left|z_{1}\right|<1$. Naming $z_{2}$ the other root, computations give:

$$
\operatorname{Res}(0, R)=-\mathrm{e}^{\mathrm{i} s}
$$

but the calculation of $\operatorname{Res}\left(z_{1}, R\right)$ is not so straightforward. Rational decomposition of $R$ is:

$$
R(z)=\frac{-\mathrm{e}^{\mathrm{i} s}}{z}+\frac{a_{1}}{z-z_{1}}+\frac{b_{1}}{\left(z-z_{1}\right)^{2}}+\frac{a_{2}}{z-z_{2}}+\frac{b_{2}}{\left(z-z_{2}\right)^{2}}
$$

where after obvious computations:

$$
\begin{aligned}
& a_{1}=\operatorname{Res}\left(z_{1}, R\right) \\
& b_{1}=\frac{\left(z_{1}^{2} \mathrm{e}^{-\mathrm{i} s}-\mathrm{e}^{\mathrm{i} s}\right)}{z_{1}\left(z_{1}-z_{2}\right)^{2}} \\
& a_{2}=\operatorname{Res}\left(z_{2}, R\right) \\
& b_{2}=\frac{\left(z_{2}^{2} \mathrm{e}^{-\mathrm{i} s}-\mathrm{e}^{\mathrm{i} s}\right)}{z_{2}\left(z_{1}-z_{2}\right)^{2}} .
\end{aligned}
$$


Multiplying (26) by $z$ and doing $z \rightarrow \infty$, we get:

$$
\mathrm{e}^{\mathrm{i} s}=a_{1}+a_{2} .
$$

Evaluating relation (26) at $z=z_{1}+z_{2}=-2 r$, and using $z_{1} z_{2}=1$ we get too:

$$
\begin{aligned}
& \frac{\left(4 r^{2} \mathrm{e}^{-\mathrm{i} s}-\mathrm{e}^{\mathrm{i} s}\right)}{-2 r\left(4 r^{2}-4 r^{2}+1\right)^{2}}=\frac{-\mathrm{e}^{\mathrm{i} s}}{-2 r}+\frac{a_{1}}{z_{2}}+\frac{b_{1}}{\left(z_{2}\right)^{2}}+\frac{a_{2}}{z_{1}}+\frac{b_{2}}{\left(z_{1}\right)^{2}} \\
& \frac{\left(4 r^{2} \mathrm{e}^{-\mathrm{i} s}-\mathrm{e}^{\mathrm{i} s}\right)}{-2 r}=\frac{\mathrm{e}^{\mathrm{i} s}}{2 r}+a_{1} z_{1}+b_{1}\left(z_{1}\right)^{2}+a_{2} z_{2}+b_{2}\left(z_{2}\right)^{2} .
\end{aligned}
$$

But successively we may write:

$$
\begin{aligned}
b_{1}\left(z_{1}\right)^{2}+b_{2}\left(z_{2}\right)^{2} & =\frac{z_{1}\left(z_{1}^{2} \mathrm{e}^{-\mathrm{i} s}-\mathrm{e}^{\mathrm{i} s}\right)}{\left(z_{1}-z_{2}\right)^{2}}+\frac{z_{2}\left(z_{2}^{2} \mathrm{e}^{-\mathrm{i} s}-\mathrm{e}^{\mathrm{i} s}\right)}{\left(z_{1}-z_{2}\right)^{2}} \\
& =\frac{1}{4\left(r^{2}-1\right)}\left(z_{1}\left(z_{1}^{2} \mathrm{e}^{-\mathrm{i} s}-\mathrm{e}^{\mathrm{i} s}\right)+z_{2}\left(z_{2}^{2} \mathrm{e}^{-\mathrm{i} s}-\mathrm{e}^{\mathrm{i} s}\right)\right) \\
& =\frac{1}{4\left(r^{2}-1\right)}\left(\left(z_{1}^{3}+z_{2}^{3}\right) \mathrm{e}^{-\mathrm{i} s}-\left(z_{1}+z_{2}\right) \mathrm{e}^{\mathrm{i} s}\right) \\
& =\frac{1}{4\left(r^{2}-1\right)}\left(\left(-8 r^{3}+6 r\right) \mathrm{e}^{-\mathrm{i} s}+2 r \mathrm{e}^{\mathrm{i} s}\right)
\end{aligned}
$$

where we use $z_{1} z_{2}=1, z_{1}+z_{2}=-2 r$ and other relations that may be deduced like

$$
z_{1}^{3}+z_{2}^{3}=\left(z_{1}+z_{2}\right)^{3}-3 z_{1}^{2} z_{2}-3 z_{2}^{2} z_{1}=-8 r^{3}-3 z_{1} z_{2}\left(z_{1}+z_{2}\right)=-8 r^{3}+6 r
$$

and

$$
\left(z_{1}-z_{2}\right)^{2}=\left(z_{1}+z_{2}\right)^{2}-4 z_{1} z_{2}=4 r^{2}-4=4\left(r^{2}-1\right) .
$$

From (28) and (29) we deduce:

$$
\begin{aligned}
a_{1} z_{1}+a_{2} z_{2} & =\frac{\left(4 r^{2} \mathrm{e}^{-\mathrm{i} s}-\mathrm{e}^{\mathrm{i} s}\right)}{-2 r}-\frac{\mathrm{e}^{\mathrm{i} s}}{2 r}-\frac{1}{4\left(r^{2}-1\right)}\left(\left(-8 r^{3}+6 r\right) \mathrm{e}^{-\mathrm{i} s}+2 r \mathrm{e}^{\mathrm{i} s}\right) \\
& =-2 r \mathrm{e}^{-\mathrm{i} s}-\frac{2 r}{4\left(r^{2}-1\right)}\left(\left(-4 r^{2}+3\right) \mathrm{e}^{-\mathrm{i} s}+\mathrm{e}^{\mathrm{i} s}\right) \\
& =-2 r\left[\mathrm{e}^{-\mathrm{i} s}+\frac{1}{4\left(r^{2}-1\right)}\left(\left(-4 r^{2}+3\right) \mathrm{e}^{-\mathrm{i} s}+\mathrm{e}^{\mathrm{i} s}\right)\right] \\
& =-\frac{2 r}{4\left(r^{2}-1\right)}\left(\mathrm{e}^{\mathrm{i} s}-\mathrm{e}^{-\mathrm{i} s}\right) .
\end{aligned}
$$

We then obtain the following system giving the residus:

$$
\left\{\begin{array}{l}
a_{1}+a_{2}=\mathrm{e}^{\mathrm{i} s} \\
a_{1} z_{1}+a_{2} z_{2}=-\frac{r}{2\left(r^{2}-1\right)}\left(\mathrm{e}^{\mathrm{i} s}-\mathrm{e}^{-\mathrm{i} s}\right) .
\end{array}\right.
$$

Solving (31), we get:

$$
\begin{aligned}
& a_{1}=\frac{1}{1-z_{1}^{2}}\left(\mathrm{e}^{\mathrm{i} s}+\frac{z_{1} r}{2\left(r^{2}-1\right)}\left(\mathrm{e}^{\mathrm{i} s}-\mathrm{e}^{-\mathrm{i} s}\right)\right) \\
& a_{2}=\frac{1}{1-z_{2}^{2}}\left(\mathrm{e}^{\mathrm{i} s}+\frac{z_{2} r}{2\left(r^{2}-1\right)}\left(\mathrm{e}^{\mathrm{i} s}-\mathrm{e}^{-\mathrm{i} s}\right)\right)
\end{aligned}
$$

and then according to (25): 


$$
\begin{aligned}
K(s, r) & =-4 \mathrm{i} \pi\left[-\mathrm{e}^{\mathrm{i} s}+\frac{1}{1-z_{1}^{2}}\left(\mathrm{e}^{\mathrm{i} s}+\frac{z_{1} r}{2\left(r^{2}-1\right)}\left(\mathrm{e}^{\mathrm{i} s}-\mathrm{e}^{-\mathrm{i} s}\right)\right)\right] \\
& =\frac{4 \mathrm{i} z_{1} \pi}{z_{1}^{2}-1}\left[z_{1} \mathrm{e}^{\mathrm{i} s}+\frac{r}{2\left(r^{2}-1\right)}\left(\mathrm{e}^{\mathrm{i} s}-\mathrm{e}^{-\mathrm{i} s}\right)\right] \\
& =\frac{2 \mathrm{i} z_{1} \pi}{\left(z_{1}^{2}-1\right)\left(r^{2}-1\right)}\left[\left(2 z_{1}\left(r^{2}-1\right)+r\right)\left(\mathrm{e}^{\mathrm{i} s}-r \mathrm{e}^{-\mathrm{i} s}\right)\right]
\end{aligned}
$$

with $z_{1}=-r+\sqrt{r^{2}-1}$ root of $z^{2}+2 r z+1$ such that $\left|z_{1}\right|<1$.

We deduce

$$
\begin{aligned}
& I(s, r)=\frac{4 z_{1} \pi}{z_{1}^{2}-1}\left[2 z_{1}+\frac{2 r}{r^{2}-1}\right] \sin (s) \\
& J(s, r)=\frac{4 \pi}{1-z_{2}^{2}} \cos (s)
\end{aligned}
$$

with $z_{2}=-r-\sqrt{r^{2}-1}$ root of $z^{2}+2 r z+1$ such that $\left|z_{2}\right|>1$.

These expressions allow us to compute numerical solutions of system of equations of synchronization $Y(y, z)=$ $Z(y, z)=0$.

\section{References}

[1] E. Absi, J. Lerbet, Instability of elastic bodies, Mechanic Research Communications 31 (1) (2004) 39-44.

[2] B. Belgacem, D. Alquier, P. Muralt, J. Baborowski, S. Lucas, R. Jerisian, Optimization of the fabrication of sealed capacitive transducers using surface micromachining, Journal of Micromechanics and Microengineering 14 (2004) 1-6.

[3] V. Bolotin, Non-Conservative Problems of the Theory of Elastic Stability, Pergamon Press, 1963.

[4] P. Gallina, About the stability of non-conservative undamped systems, Journal of Sound and Vibration 262 (2003) 977-988; Journal of Applied Mathematics and Mechanics 64 (6) (2000) 895-902.

[5] A.N. Kounadis, On the failure of static stability analyses of non-conservative systems in regions of divergence instability, International Journal of Solids and Structures 31 (1994) 2099-2120.

[6] H. Leipholz, Stability Theory, J. Wiley, 1987.

[7] R.M. Lin, W.J. Wang, Structural dynamics of microsystems-current state of research and future directions, Mechanical Systems and Signal Processing 20 (2006) 1015-1043.

[8] F.C. Moon, Chaotic Vibrations, J. Wiley, 2003.

[9] Nguyen Quoc Son, Stabilité des structures élastiques, Springer-Verlag, 1995.

[10] V. Rochus, D.J. Rixen, J.-C. Golinval, Electrostatic coupling of MEMS structures: Transient simulations and dynamic pull-in, Nonlinear Analysis 63 (2005) e1619-e1633.

[11] M. Roseau, Vibrations des systèmes mécaniques, Masson, 1984

[12] J. Teva, G. Abadal, Z.J. Davis, J. Verd, X. Borrise, A. Boisen, F. Pérez-Murano, N. Barniol, On the electromechanical modelling of a resonating nano-cantilever-base transducer, Ultramicroscopy 100 (2004) 225-232.

[13] M.I. Younis, A.H. Nayfeh, A study of nonlinear response of a resonant microbeam to an electric actuation, Nonlinear Dynamics 31 (2003) 91-117. 